\title{
Clinical Usefulness of HLA-B*58:01 Genotyping in Gouty Arthritis
}

\author{
Carrel Ka Lung $\mathrm{Yu}^{\star}$, Chi Chiu Mok \\ Department of Medicine, Tuen Mun Hospital, Hong Kong SAR, China
}

\begin{abstract}
Allopurinol is an effective urate lowering agent but may lead to rare but life-threatening severe cutaneous adverse reactions (SCAR). Genetic predisposition, age, sex, renal function, dosage and concomitant diuretic use are known risk factors of allopurinol related SCAR. Among these factors, HLA-B ${ }^{\star} 58: 01$ confers the highest risk. Frequency of the HLA-B ${ }^{\star}$ 58:01 allele varies significantly across different ethnic groups. Although the usefulness of HLA-5801 genotyping before initiation of allopurinol has been confirmed by clinical studies, its positive predictive value for SCAR is low because of the low prevalence of HLA-5801 in some localities. Thus, health economic analysis does not consistently show cost-effectiveness of universal screening of HLA-5801 before initiation of allopurinol. However, screening of this genotype in high-risk subjects, including those with renal impairment or advanced age, should be considered on individual basis.
\end{abstract}

Keywords: HLA-B`58:01; Allopurinol; Hypersensitivity; Severe Cutaneous Adverse Reactions; Cost-Effectiveness; Genetic Screening.

\section{INTRODUCTION}

With ageing, change in lifestyle and diet, the burden of gouty arthritis has been increasing. The prevalence of gout in China increased from $0.15 \%$ in the 1980 s to $1.98 \%$ in the $2000 \mathrm{~s}$ [1]. The highest prevalence of gout was found in Māori tribes (6.7\%) and in Taiwan aborigines (11.7\%) [2].

Gouty arthritis is related to formation and deposition of monosodium urate (MSU) crystals in the joints and soft tissues. The formation of MSU crystal is closely linked with serum concentration of uric acid. Crystallization occurs when this is above $0.40 \mathrm{mmol} / \mathrm{L}$, which corresponds to the supersaturation level [3]. Crystal deposition is reversible, and crystals can dissolve when the concentration of serum uric acid (SUA) is reduced to levels $0.36 \mathrm{mmol} / \mathrm{L}$ [4]. Therefore, the European League Against Rheumatism (EULAR) and the American College of Rheumatology (ACR) recommend uratelowering therapy to a target SUA level of $0.36 \mathrm{mmol} / \mathrm{L}$ (or $6 \mathrm{mg} / \mathrm{dl}$ ) or less in the treatment of gout.

Allopurinol is a xanthine oxidase inhibitor and an effective urate-lowering drug. Since the drug was first marketed in 1966, allopurinol has been the most commonly used drug for the treatment of gout. Although it is well tolerated in most patients, minor maculopapular eruption may develop in around $2 \%$ of patients, in whom around 5\% have to discontinue treatment [5]. Moreover, the drug is associated with severe cutaneous adverse reaction (SCAR) in a minority of users. Despite this, with the increasing prevalence of gout worldwide, prescription of allopurinol and reports of severe hypersensitivity reactions are on a rising trend.

\section{ALLOPURINOL ASSOCIATED SEVERE CUTANEOUS ADVERSE REACTION (SCAR)} Allopurinol associated SCAR is a potentially fatal condition. This includes Steven-Johnson syndrome/ toxic epidermal necrolysis (SJS/TEN), drug reaction with eosinophilia and systemic symptoms (DRESS) and allopurinol hypersensitivity syndrome (AHS). A large cohort study reported the incidence of allopurinol associated SCAR to be 0.69 per 1000 person-years (95\% CI 0.50-0.92) [6].

() 2019 by the Hong Kong Society of Rheumatology and World Scientific Publishing Co. Pte. Ltd. ə Open Access article under the CC BY-NC-ND license (http://creativecommons.org/licenses/by-nc-nd/4.0/).

Received 4 April 2019; Accepted 30 May 2019; Published 5 July 2019

*Corresponding author: Carrel K. L. Yu, Department of Medicine, Tuen Mun Hospital, Tsing Chung Koon Rd, Tuen Mun, Hong Kong, Email: dryukalung@ gmail.com; carrelyu@yahoo.com 
Allopurinol associated SCAR typically occurs in the first few weeks or months after starting allopurinol. The median time of onset of this severe reaction was 3 weeks and $90 \%$ of cases occurred within the first 8-9 weeks [7]. The presence of the HLA-B*58:01 allele was strongly associated with allopurinol hypersensitivity with hazard ratios in excess of 100 [8]. Other important predisposing factors for allopurinol related SCAR include female sex, advanced age, drug concentration, drug dosage, chronic kidney disease and concomitant diuretic use.

A Taiwanese population-based study showed that females were at higher risk for allopurinol hypersensitivity (OR 1.45; 95\% CI, 1.35-1.56; P < 0.001) and related mortality (OR 1.63; 95\% CI, 1.28-2.08; $\mathrm{P}<0.001)$. Moreover, the incidence of allopurinol hypersensitivity increased significantly in new users above 60 years of age (incidence per 1,000 new users 3.23 at $0-39$ years, 3.46 at $40-59$ years, 5.73 at $60-79$ years, 9.81 at 80 years or above), particularly above 80 years of age (OR 2.27; 95\% CI, 1.97-2.60; P < 0.001). The risk of allopurinol hypersensitivity-related mortality was 12fold higher in patients older than 80 years (OR 12.37; 95\% CI, 6.24-24.53; P < 0.001) compared with those below 40 years of age [9]. Hande et al. demonstrated that a higher starting dose was associated with a higher incidence of allopurinol hypersensitivity reaction. The mean starting dose $(183.5+/-14.0 \mathrm{mg} /$ day $)$ in those who developed allopurinol hypersensitivity was significantly higher than those who did not develop this reaction $(112.2+/-6.3 \mathrm{mg} /$ day; $\mathrm{P}<0.001)$. Patients treated with a higher allopurinol starting dose than the creatinine clearance-based dose were more likely to develop hypersensitivity reaction than controls (OR 16.7; 95\% CI 5.7-47.6, $\mathrm{P}<0.001)$. On the other hand, patients with the same or lower than the creatinine clearance-based dose had less chance of developing hypersensitivity (OR 6.9, 95\% CI 2.9-16.5, P < 0.001) [10]. Stamp et al. performed a retrospective case-control study which determined the relationship between allopurinol dosing and hypersensitivity reaction matched with sex, age and estimated glomerular filtration rate (eGFR) [11]. In the highest quintile of starting dose per estimated GFR at $>4.05 \mathrm{mg} / \mathrm{ml} / \mathrm{min} / 1.73 \mathrm{~m}^{2}$, the odds ratio of hypersensitivity was 23.2 compared with starting dose at $<1.25 \mathrm{mg} / \mathrm{ml} / \mathrm{min} / 1.73 \mathrm{~m}^{2}(\mathrm{P}<0.001)$. It was further estimated that the safe starting dosage of allopurinol was $1.5 \mathrm{mg}$ per estimated GFR. This study provides the basis for eGFR-based allopurinol starting dose to reduce the risk of severe hypersensitivity reaction. With
Table 1. Recommended starting dose of allopurinol according to estimated GFR by Stamp et al. [9].

\begin{tabular}{lc}
\hline $\begin{array}{l}\text { Estimated GFR }(\mathbf{m l} / \mathbf{m i n u t e} / \\
\left.\mathbf{1 . 7 3} \mathbf{~}^{2}\right)\end{array}$ & $\begin{array}{l}\text { Starting dose of allopurinol } \\
(\mathbf{m g})\end{array}$ \\
\hline $31-45$ & 50 \\
$61-90$ & 100 \\
$91-130$ & 150 \\
$>130$ & 200 \\
\hline
\end{tabular}

an estimated GFR of $31-45,61-90,91-130$ and $>130$ $\mathrm{ml} /$ minute $/ 1.73 \mathrm{~m}^{2}$ respectively, allopurinol should be started at 50,100, 150 and $200 \mathrm{mg} /$ day (Table 1).

Allopurinol is metabolized to oxypurinol after ingestion. Lymphocyte transformation test suggested that it is oxypurinol which causes the adverse skin reaction [12]. Oxypurinol has a longer half-life than allopurinol. However, the threshold oxypurinol concentration that is associated with excess risk of hypersensitivity reaction is not certain. In patients with impaired renal function, elimination of the allopurinol metabolite is impaired and the risk of hypersensitivity is higher because of the higher serum concentration. It is reported that the chance of development of allopurinol hypersensitivity in chronic kidney disease (CKD) patients are three to four times higher than those with normal renal function [13]. This was echoed by another study which showed a similar increased risk of allopurinol hypersensitivity in CKD patients (odds ratio of 4.7, 95\% CI 2.3-9.3; P < 0.0001) [14]. Diuretics alter the function of the kidney tubules to process sodium and urate, which in turn increases the level of serum urate. Furosemide interacts with allopurinol in a complicated manner that results in attenuation of the hypouricaemic effects of oxypurinol. It increases serum concentration not only of urate but also of oxypurinol [15].

\section{HLA GENOTYPE AND ALLOPURINOL} ASSOCIATED SCAR

Recent genomic research has identified genes that confer risk for drug specific SCARs. Studies found that there is an association between human leukocyte antigen (HLA) and the development of hypersensitivity reaction in patients exposed to allopurinol. The immune system is modulated by the HLA system which plays a key role in determining susceptibility to infection and 
autoimmune diseases. The amino acid sequence of HLA molecules determines peptide binding and antigen presentation to T lymphocytes. Several mechanisms of immune mediated adverse drug reaction are proposed. For allopurinol associated SCAR, it is suggested the drug binds to the HLA molecules which alter the binding cleft. The altered repertoire of self-peptides is then recognized and presented to T cells [16]. Oxypurinol binds with a high affinity to the HLA-B ${ }^{\star} 58: 01$ molecule, therefore individuals with the HLA-B ${ }^{\star}$ 58:01 allele exhibit more pronounced $\mathrm{T}$ cell response to oxypurinol [17]. The presence of the HLA-B ${ }^{\star} 58: 01$ allele and a high concentration of oxypurinol function synergistically to increase the number of potentially immunogenicpeptide-oxypurinol-HLA-B ${ }^{\star}$ 58:01 complexes on cell surface, increasing the risk of T-cell sensitization and a subsequent hypersensitivity reaction [18].

\section{DIAGNOSTIC UTILITY OF HLA-B*58:01 GENOTYPING}

The prevalence of the HLA-B ${ }^{\star} 58: 01$ allele is reported from $<1 \%$ in Caucasians to up to $8 \%$ in Southeast Asians [19]. It is more commonly found in Koreans and Han Chinese. Because of the variation in the frequency of this allele, the incidence of allopurinol associated SCAR also varies across different populations (Table 2).
Hung et al. compared 51 allopurinol associated SCAR patients with 135 patients exposed to allopurinol for 6 months but without adverse events and 93 subjects from the general population in Taiwan [14]. It was reported that all the $51(100 \%)$ patients with SCAR were positive for the HLA-B ${ }^{\star 58: 01}$ allele. Chronic renal insufficiency was associated with increased risk of allopurinol induced SCAR with an odds ratio of 4.7 (95\% CI 2.3-9.3) [14]. In another study from Thailand, Tassaneeyahul et al. [20] examined 27 allopurinol associated SJS/TEN and 54 allopurinol tolerant patients. All of the $27(100 \%)$ allopurinol-induced SJS/TEN patients carried the HLA-B ${ }^{\star 58: 01}$ as compared to only 7 (12.96\%) of the control patients (odds ratio $348.3,95 \% \mathrm{CI}$ $\left.19.2-6336.9, \mathrm{P}=1.6 \times 10^{-13}\right)$. The sensitivity and specificity of the HLA-B ${ }^{\star 58: 01}$ allele for prediction of allopurinolinduced SJS/TEN were 100 and 87\%, respectively. Assuming a $0.2 \%$ prevalence rate of allopurinol related SCAR, the positive and negative predictive value of the HLA- ${ }^{\star} 58: 01$ allele for this serious reaction was $1.52 \%$ and $100 \%$, respectively [20]. In Korea Kang et al. [21] examined 25 cases of allopurinol-induced SCARs (20 cases of drug-induced hypersensitivity syndrome and five cases of Stevens-Johnson syndrome/toxic epidermal necrolysis) and 57 patients tolerant to allopurinol. Frequencies of HLA-B ${ }^{\star 58: 01}$ were significantly higher

Table 2. Risk of allopurinol induced Stevens-Johnson syndrome and toxic epidermal necrolysis in subjects with HLA-B ${ }^{\star}$ 58:01 allele.

\begin{tabular}{|c|c|c|c|c|c|c|}
\hline \multirow[t]{2}{*}{ Author, publication year } & \multirow[t]{2}{*}{ Region } & \multicolumn{2}{|l|}{ Case } & \multicolumn{2}{|l|}{ Control } & \multirow[t]{2}{*}{ Odds ratio $[95 \% \mathrm{CI}]$} \\
\hline & & Events & Total & Events & Total & \\
\hline Huang, 2005 & Taiwan & 21 & 21 & 19 & 93 & $164.28[9.52-2833.92]$ \\
\hline Kaniwa, 2008 & Japan & 4 & 20 & 6 & 986 & $40.83[10.50-158.80]$ \\
\hline Loujou, 2008 & Europe & 15 & 27 & 28 & 1822 & 80.09 p34.37-186.61] \\
\hline Tassaneeyakul, 2009 & Thailand & 27 & 27 & 63 & 400 & $292.32[17.60-4854.06]$ \\
\hline Kang, 2011 & Korean & 4 & 5 & 59 & 485 & $28.88[3.17-262.79]$ \\
\hline Cristallo, 2011 & Italy & 3 & 7 & 6 & 115 & $13.6[2.46-75.16]$ \\
\hline Lee, 2012 & Australia & 5 & 6 & 13 & 134 & $46.54[5.04-429.32]$ \\
\hline Cao, 2012 & China & 13 & 13 & 80 & 572 & $165.19[9.72-2806.13]$ \\
\hline Tohkin, 2013 & Japan & 10 & 18 & 6 & 493 & $101.5[29.66-347.06]$ \\
\hline Chiu, 2012 & Hong Kong & 13 & 14 & 81 & 569 & $78.32[10.11-606.87]$ \\
\hline Goncalo, 2013 & Portugal & 4 & 6 & 63 & 3200 & $99.59[17.91-553.71]$ \\
\hline Cheng, 2015 & China & 49 & 51 & 10 & 99 & $218.1[45.92-1035.30]$ \\
\hline Ng, 2016 & Taiwan & 21 & 25 & 139 & 710 & $21.57[7.29-63.84]$ \\
\hline
\end{tabular}


in SCARs compared with tolerant controls, $92.0 \%$ and $10.5 \%$ (odds ratio $97.8, \mathrm{P}=2.45 \times 10^{-11}$ ) [22]. The association of HLA-B ${ }^{\star} 58: 01$ with allopurinol associated SCAR is less pronounced in the European population. In a European study (RegiSCAR), HLA-B genotyping was performed on 150 patients of SJS and TEN. Fifty percent of Europeans who developed the hypersensitivity carried the HLA-B ${ }^{\star} 58: 01$ allele (odds ratio 80 , CI 34-187, $\mathrm{P}<10^{-6}$ ) [22]. Taken together, it is apparent that in Asian populations, there is stronger association between the HLA-B ${ }^{\star} 58: 01$ allele and risk of SCAR.

The association between HLA-B ${ }^{\star} 58: 01$ and the risk of allopurinol associated SCAR was also observed in Hong Kong, where $92 \%$ of the population is Han Chinese [23]. In a case-control study, HLA-B ${ }^{\star 58: 01}$ genotyping was performed for 20 allopurinol-induced SCAR and 30 allopurinol tolerant patients [24]. Nineteen patients with allopurinol-induced SCAR (95\%), carried HLA-B ${ }^{\star 58: 01}$. In contrast, only four (13\%) of the control patients carried the allele. The positive rate of the HLA-B ${ }^{\star 58: 01}$ was significantly higher in allopurinol-induced SCAR patients with an odds ratio of 123.5 (95\% CI 12.8-1195.1, $\mathrm{P}<1 \times 10^{-4}$ ). The sensitivity and specificity of the HLA-B ${ }^{\star} 58: 01$ allele for prediction of allopurinol-induced SCAR were $100 \%$ and $86.7 \%$, respectively [24].

\section{COST-EFFECTIVENESS OF HLA-B*58:01}

\section{GENOTYPING AS SCREENING}

Astheallele frequency of HLA-B ${ }^{\star}$ 58:01 is highly variable among different ethnic groups, cost-effectiveness must be taken into account when HLA-B ${ }^{\star} 58: 01$ is used as a screening test. A number of studies were conducted to examine universal HLA-B ${ }^{\star} 58: 01$ screening before allopurinol use. The first study was conducted in Thailand. In the Thai population, the incidence of allopurinol associated SJS/TEN is 0.0016 [25]. The risk of allopurinol-induced SJS/TEN was significantly greater in patients with HLA-B ${ }^{\star} 58: 01$ when compared with those who did not carry this allele, with an odds ratio of 348.3 (95\% CI 19.2-6336.9, $\mathrm{P}=1.6 \times 10^{-13}$ ). In the analysis, genetic testing for HLA-B ${ }^{\star 58: 01}$ before allopurinol use was expected to lead to a decrease in incidence of SJS/TEN 1.57 cases per 1,000 exposure and a decrease in incidence of attributable death of 0.18 cases per 1,000 exposure. Based on a hypothetical cohort of 1,000 patients at age 30 years, the incremental total cost was USD 29,804 and incremental qualityadjusted life-year (QALY) was 5.89 with an incremental cost-effectiveness ratio (ICER) of USD 5,062 per QALY gained. The genetic testing for HLA-B ${ }^{\star} 58: 01$ was shown to be cost-effective. It should be noted that subjects who were identified as positive for the HLA-B $^{\star}$ 58:01 allele received probenecid, rather than febuxostat, as an alternative drug. In addition, the utility score was extrapolated from another study conducted in Europe since there was no such data for Thai patients with gout.

However, similar analysis in the Malaysian population was not that promising. A study by Chong et al. evaluated the use of HLA-B ${ }^{\star} 58: 01$ screening before allopurinol initiation in a hypothetical cohort of 10,000 patients with chronic gout aged 50 years with normal renal function [26]. Three treatment strategies were tested, including (1) allopurinol $300 \mathrm{mg} /$ day administered to all patients without HLA-B ${ }^{\star 58: 01}$ screening; (2) HLA-B ${ }^{\star 58: 01}$ screening conducted before allopurinol initiation; and (3) probenecid prescribed as the first-line agent without HLA- ${ }^{\star} 58: 01$ screening. Compared with the first strategy, HLA-B ${ }^{\star} 58: 01$ allele screening before allopurinol initiation resulted in 0.252 QALYs loss per patient at an additional cost of USD 879. However, probenecid prescribing resulted in 1.928 QALY loss per patient at an additional cost of USD 6848. Such a finding was related to the low incidence of allopurinol-induced SJS/TEN in Malaysia. The study suggests that neither HLA-B ${ }^{\star}$ 58:01 screening nor probenecid prescribing is likely to be cost-effective compared with current practice in Malaysia. Variation in HLA-B ${ }^{\star}$ 58:01 gene frequency in this multi-ethnic country may also affect the cost-effectiveness.

While universal screening for all patients with allopurinol treatment may not be feasible, the costeffectiveness of HLA-B ${ }^{\star}$ 58:01 screening was re-evaluated in selected patients in Singapore [27]. Six strategies, including those that incorporate HLA- ${ }^{\star} 58$ :01 genetic testing and/or enhanced safety programs, to reduce the risk of allopurinol-induced SJS/TEN and to identify the conditions in which each strategy is incrementally costeffective from the Singapore health system perspective were evaluated. It was concluded that HLA-B ${ }^{\star 58: 01}$ genetic testing for all gout patients commencing urate lowering therapy reduced the overall QALYs at a population level if used to avoid allopurinol associated SCAR in all HLA-B ${ }^{\star 58: 01-p o s i t i v e ~ p a t i e n t s . ~ B a s e d ~}$ on the threshold of US\$50,000 per QALY, all three strategies involving HLA-B ${ }^{\star}$ 58:01-guided urate lowering therapy selection were not cost-effective. Therefore, 
universal screening before allopurinol treatment is not recommended in Singapore.

Plumpton et al. examined prospective testing for HLA-B ${ }^{\star} 58: 01$ as a strategy to prevent serious adverse reactions to allopurinol in the National Health Service in the UK [28]. The number of patients (needed for testing) to prevent one case of adverse drug reaction was 11,286 (95\% central range (CR) 2,573-53,594). Cost and QALY gains were small, $£ 103$ (95\% CR, £98-£106) and 0.0023 (95\% CR, $-0.0006-0.0055)$, respectively, resulting in an ICER of $£ 44,954$ per QALY gained. The probability of testing being cost-effective at a threshold of $£ 30,000$ per QALY was 0.25 . The ICER for patients with chronic renal insufficiency was $£ 38,478$ per QALY gained. The finding did not support routine testing for HLA- ${ }^{\star}{ }^{58: 01}$ in order to reduce the incidence of adverse drug reactions in patients being prescribed allopurinol for gout in the UK [28].

These studies show inconsistent results regarding the cost-effectiveness of using HLA-B ${ }^{\star} 58$ :01 genotyping as a screening test before the commencement of allopurinol. Whilst the negative predictive value of the test is high, the positive predictive value is low. This is because the prevalence of HLA-B ${ }^{\star 58: 01}$ allele is low in the general population. While universal screening may not be cost-effective, testing for high risk groups may be of value. Park et al. evaluated HLA-B ${ }^{\star 58: 01}$ genotyping for the treatment of gout in patients with chronic renal insufficiency in Korea [29]. It was found that the total expected cost and probability of continuation of gout treatment without SCARs for the conventional and HLA-B ${ }^{\star} 58: 01$ screening strategies were $\$ 1,193$ and $97.8 \%$, and $\$ 1,055$ and $100 \%$, respectively. The results suggested that HLA-B ${ }^{\star} 58: 01$ genotyping was less costly and more effective than treatment without genotyping, and HLA-B ${ }^{\star 58: 01}$ genotyping could considerably reduce the occurrence of allopurinol-induced SCARs and related deaths. This finding supports the view that HLA-B ${ }^{\star 58: 01}$ genotyping should be considered for patients with chronic renal insufficiency.

In the US, the SJS/TEN risks and HLA-B ${ }^{\star 58: 01}$ prevalence were $1 / 3846$ and $0.7 \%$ among Caucasians and Hispanics, $1 / 735$ and $3.8 \%$ among African Americans, and $1 / 336$ and $7.4 \%$ among Asians, respectively. Jutkowitz et al. determined the value of universal testing for HLA-B ${ }^{\star 58: 01}$ compared to no testing prior to the initiation of allopurinol in various ethnic groups. Those who tested positive for HLA- ${ }^{\star} 58: 01$ received febuxostat. Compared to no
Table 3. Frequencies of HLA-B ${ }^{\star} 58: 01$

allele in different regions of the world.

\begin{tabular}{lc}
\hline Country & HLA-B $\mathbf{5 8 : 0 1}$ allele frequency \\
\hline Singapore & $20.1 \%$ \\
Taiwan & $20.0 \%$ \\
Hong Kong & $9.0 \%$ \\
China & $12.0 \%$ \\
Korea & $12.0 \%$ \\
Thailand & $7.7 \%$ \\
Italy & $6.7 \%$ \\
Portugal & $2.6 \%$ \\
\hline
\end{tabular}

testing, universal testing for HLA-B ${ }^{\star} 58$ :01 cost more and was more effective for all ethnicities. The ICERs varied substantially across ethnic groups, due to the different HLA-B ${ }^{\star 58: 01}$ prevalence. HLA-B ${ }^{\star 58: 01}$ testing was cost-effective for African Americans (ICER \$83,450) and Asians (ICER \$64,190), but not for Caucasians or Hispanics (ICER \$183,720), using a threshold of \$109,000/QALY [30].

Because of differences in prevalence of HLA- $B^{\star} 58: 01$, risk of SCAR associated with HLA-B ${ }^{\star} 58: 01$ allele and medical expenses, the cost-effectiveness of HLA-B ${ }^{\star} 58: 01$ genetic screening varies greatly across different health care systems (Table 3). There is no consensus whether such a screening strategy should be adopted at the societal level. However, current data suggest screening in selected patients, particularly patients with renal impairment may be more cost-effective. Age is another important risk factor for allopurinol associated SCAR. However, current costeffective studies do not analyse the effect of age. A Taiwanese study reported that age above 80 significantly increased the risk [9]. It is sensible to consider offering HLA-5801 genotyping for subjects at or above 80 years of age.

\section{CONCLUSION}

Allopurinol remains the most frequently used urate lowering drug for the treatment of gouty arthritis. Although it is effective and inexpensive, it may cause rare but life-threatening hypersensitivity reactions. Therefore, allopurinol should be used judiciously and the current practice in most localities is not to use it in patients with asymptomatic hyperuricaemia until more research data are available.

It is likely that the risk of allopurinol associated SCAR is dose-dependent. Therefore, it is prudent to 
adopt the "start low, go slow" approach for all patients, particularly those with increased risk of developing allopurinol hypersensitivity. Starting dose should be adjusted according to the estimated GFR.

Although HLA-B ${ }^{\star} 58: 01$ is strongly associated with SCAR, the cost-effectiveness of universal screening of this genotype before initiation of allopurinol remains controversial. Screening of HLA-B ${ }^{\star 58: 01}$ in high risk individuals is a more pragmatic approach. It is justifiable to perform HLA-B ${ }^{\star}$ 58:01 genotyping for patients with CKD or aged above 80 years prior to initiation of allopurinol. Alternative urate lowering therapy should be considered if the HLA-B ${ }^{\star 58: 01}$ allele is present. Finally, patient education for prompt cessation of allopurinol in case of any skin rash development may reduce the severity of SCAR if it happens.

\section{REFERENCES}

[1] Zeng Q, Chen R, Darmawan J, Xiao Z, Chen S, Wigley R, et al. Rheumatic diseases in China. Arthritis Res Ther. 2008; 10(1):R17.

[2] Klemp P, Stansfield S, Castle B, Robertson M. Gout is on the increase in New Zealand. Ann Rheum Dis. 1997;56(1):22-6.

[3] Martillo MA, Nazzal L, Crittenden DB. The crystallization of monosodium urate. Curr Rheumatol Rep. 2014;16(2):400.

[4] Li-Yu J, Clayburne G, Sieck M, et al. Treatment of chronic gout. Can we determine when urate stores are depleted enough to prevent attacks of gout? J Rheumatol. 2001;28(3):577-80.

[5] McInnes G, Lawson D, Jick H. Acute adverse reactions attributed to allopurinol in hospitalised patients. Ann Rheum Dis. 1981;40(3):245-9.

[6] Kim S, Newcomb C, Margolis D, Roy J, Hennessy S. Severe cutaneous reactions requiring hospitalization in allopurinol initiators: a population-based cohort study. Arthritis Care Res. 2013;65(4):578-84.

[7] Ramasamy S, Korb-Wells C, Kannangara D, Smith M, Wang N, Roberts D, et al. Allopurinol hypersensitivity: a systematic review of all published cases, 1950-2012. Drug Safety. 2013;36(10):953-80.

[8] Khanna D, Fitzgerald J, Khanna P, Bae S, Singh M, Neogi T, et al. 2012 American College of Rheumatology guidelines for management of gout. Part 1: Systematic nonpharmacologic and pharmacologic therapeutic approaches to hyperuricemia. Arthritis Care Res. 2012;64(10):1431-46.

[9] Yang C, Chen C, Deng S, Huang C, Lin Y, Chen Y, et al. Allopurinol use and risk of fatal hypersensitivity reactions. JAMA Intern Med. 2015;175(9):1550.

[10] Hande K, Noone R, Stone W. Severe allopurinol toxicity. Description and guidelines for prevention in patients with renal insufficiency. J Urol. 1984;131(6):1258-9.

[11] Stamp L, Taylor W, Jones P, Dockerty J, Drake J, Frampton C, et al. Starting dose is a risk factor for allopurinol hypersensitivity syndrome: a proposed safe starting dose of allopurinol. Arthritis Rheum. 2012;64(8):2529-36.
[12] Pichler W, Tilch J. The lymphocyte transformation test in the diagnosis of drug hypersensitivity. Allergy. 2004;59(8): 809-20.

[13] Khanna D, Fuldeore MJ, Meissner BL, et al. The incidence of allopurinol hypersensitivity syndrome: a population perspective. Arthritis Rheumatol. 2008:60(suppl 542).

[14] Hung S, Chung W, Liou L, Chu C, Lin M, Huang H, et al. HLA-B ${ }^{\star 58: 01}$ allele as a genetic marker for severe cutaneous adverse reactions caused by allopurinol. Proc Natl Acad Sci. USA 2005;102(11):4134-9.

[15] Stamp L, Barclay M, O’Donnell J, Zhang M, Drake J, Frampton $\mathrm{C}$, et al. Furosemide increases plasma oxypurinol without lowering serum urate-a complex drug interaction: implications for clinical practice. Rheumatology. 2012;51(9):1670-6.

[16] Illing P, Vivian J, Dudek N, Kostenko L, Chen Z, Bharadwaj M, et al. Immune self-reactivity triggered by drug-modified HLApeptide repertoire. Nature. 2012;486(7404):554-8.

[17] Yun J, Mattsson J, Schnyder K, Fontana S, Largiadèr C, Pichler $\mathrm{W}$, et al. Allopurinol hypersensitivity is primarily mediated by dose-dependent oxypurinol-specific T cell response. Clin Exp Allergy. 2013;43(11):1246-55.

[18] Fabian H, Huser H, Narzi D, Misselwitz R, Loll B, Ziegler A, et al. HLA-B27 subtypes differentially associated with disease exhibit conformational differences in solution. J Mol Biol. 2008;376(3):798-810.

[19] Somkrua R, Eickman EE, Saokaew S, Lohitnavy M, Chaiyakunapruk N. Association of HLA-B ${ }^{\star} 5801$ allele and allopurinol-induced Stevens Johnson syndrome and toxic epidermal necrolysis: a systematic review and meta-analysis. BMC Med Genet. 2011;12:118.

[20] Tassaneeyakul W, Jantararoungtong T, Chen P, Lin P, Tiamkao $S$, Khunarkornsiri $U$, et al. Strong association between HLA-B ${ }^{\star}$ 58:01 and allopurinol-induced Stevens-Johnson syndrome and toxic epidermal necrolysis in a Thai population. Pharmacogenet Genom. 2009;19(9):704-9.

[21] Kang H, Jee Y, Kim Y, Lee C, Jung J, Kim S, et al. Positive and negative associations of HLA class I alleles with allopurinolinduced SCARs in Koreans. Pharmacogenet Genom. 2011;21(5):303-7.

[22] Lonjou C, Borot N, Sekula P, Ledger N, Thomas L, Halevy S, et al. A European study of HLA-B in Stevens-Johnson syndrome and toxic epidermal necrolysis related to five high-risk drugs. Pharmacogenet Genom. 2008;18(2):99-107.

[23] Hong Kong 2016 Population By-census Report.

[24] Chiu M, Hu M, Ng M, Yeung C, Chan J, Chang M, et al. Association between HLA-B ${ }^{\star}$ 58:01 allele and severe cutaneous adverse reactions with allopurinol in Han Chinese in Hong Kong. Br J Dermatol. 2012;167(1):44-9.

[25] Saokaew S, Tassaneeyakul W, Maenthaisong R, Chaiyakunapruk N. Cost-effectiveness analysis of HLA-B ${ }^{\star 58: 01}$ testing in preventing allopurinol-induced SJS/TEN in Thai population. PLoS ONE. 2014;9(4):e94294.

[26] Chong H, Lim Y, Prawjaeng J, Tassaneeyakul W, Mohamed Z, Chaiyakunapruk N. Cost-effectiveness analysis of HLA-B ${ }^{\star} 58$. Pharmacogenet Genom. 2018;28(2):56-67.

[27] Dong D, Tan-Koi W, Teng G, Finkelstein E, Sung C. Costeffectiveness analysis of genotyping for HLA-B ${ }^{\star} 58: 01$ and an 
enhanced safety program in gout patients starting allopurinol in Singapore. Pharmacogenomics. 2015;16(16):1781-93.

[28] Plumpton C, Alfirevic A, Pirmohamed M, Hughes D. Costeffectiveness analysis of HLA-B ${ }^{\star} 58: 01$ genotyping prior to initiation of allopurinol for gout. Rheumatology. 2017;56(10): 1729-39.

[29] Park D, Kang J, Lee J, Lee K, Wen L, Kim T, et al. Costeffectiveness analysis of HLA-B*58:01 genotyping in the treatment of gout patients with chronic renal insufficiency in Korea. Arthritis Care Res. 2015;67(2):280-7.

[30] Jutkowitz E, Dubreuil M, Lu N, Kuntz K, Choi H. The costeffectiveness of HLA-B ${ }^{\star 58: 01}$ screening to guide initial uratelowering therapy for gout in the United States. Seminars in Arthritis Rheum. 2017;46(5):594-600. 